\title{
ENDOGAMIA EN UN SINTÉTICO DE MAÍZ FORMADO CON FAMILIAS DE AUTO HERMANOS (LÍNEAS S $S_{1}$ )
}

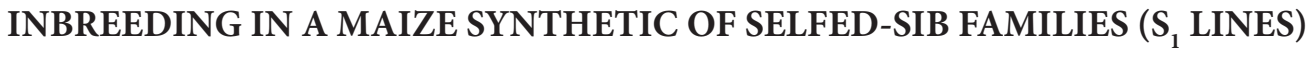

\author{
Fidel Márquez-Sánchez
}

Centro Regional Universitario Occidente, Universidad Autónoma Chapingo. Rosario Castellanos 2332, Colonia Residencial la Cruz. 44950, Guadalajara, Jalisco.

${ }^{*}$ Autor para correspondencia (fidelmqz@hotmail.com)

\section{RESUMEN}

\begin{abstract}
Los sintéticos son poblaciones mejoradas que se recomiendan a los agricultores que no pueden comprar híbridos. En el presente manuscrito se presenta el cálculo de la endogamia en un sintético de maíz (Zea mays $\mathrm{L}$.) formado con familias de auto hermanos $(\mathrm{AH})$ o líneas $\mathrm{S}_{1}$. El sintético se obtiene desde la primera generación mediante polinización libre de un compuesto balanceado de las familias. La endogamia en la primera generación se calcula mediante una ecuación que incluye a varias generaciones: $\mathrm{F}(\mathrm{AH})_{1}=(1 / 4 n m)\left[(2 m+1)+4 m(n-1) \mathrm{F}_{1}+\right.$ $\left.2(m-1) \mathrm{F}_{0}+\mathrm{F}_{-1}\right]$, en donde $n$ es el número de familias, $m$ el número de plantas por familia, y $\mathrm{F}$ es el valor del coeficiente de endogamia cuyos subíndices son las generaciones. Después de eliminar de la ecuación recurrente los términos cuya endogamia es igual a cero, el valor de la endogamia en el sintético $F_{1}$ con 10 familias y 10 de plantas por familia, fue igual a 0.0526. El número de familias influye más en la endogamia del sintético que el número de plantas por familia.
\end{abstract}

Palabras clave: Zea mays, endogamia, familias de auto hermanos, sintético.

\section{SUMMARY}

Synthetics are genetically improved populations for farmers that cannot buy hybrid seed. In this paper, inbreeding is calculated for a maize synthetic (Zea mays L.) created with selfed-sib families $(\mathrm{AH})$ or $\mathrm{S}_{1}$ lines. The synthetic is obtained from the first generation by open pollination of a balanced composite made with the families. Inbreeding in the first generation is calculated through an equation that includes several generations: $\mathrm{F}(\mathrm{AH})_{1}=(1 / 4 n m)[(2 m+1)+4$ $\left.m(n-1) \mathrm{F}_{1}+2(m-1) \mathrm{F}_{0}+\mathrm{F}_{-1}\right]$, where $n$ is the number of families, $m$ the number of plants per family, and $F$ is the inbreeding coefficient which sub-indexes are the generation numbers. After elimination of the terms with zero inbreeding in the recurrent equation, inbreeding in an $F_{1}$ synthetic with 10 families and 10 plants per family is equal to 0.0526 . The number of families affects more the synthetic inbreeding than the number of plants per family.

Index words: Zea mays, inbreeding, selfed-sib families, synthetic variety.

\section{INTRODUCCIÓN}

Las variedades sintéticas, o sintéticos, son poblaciones mejoradas de maíz (Zea mays L.) que se pueden obtener mediante más de un método de mejora genética. Dado que el costo de la semilla híbrida representa en ocasiones has- ta $15 \%$ del total de los costos de producción, no siempre los agricultores de bajos recursos pueden comprar ese tipo de semilla, por lo que prefieren sembrar poblaciones de las cuales puedan ellos mismos obtener su propia semilla. En este contexto están situados los sintéticos, ya que a los agricultores les basta, con unos cuidados mínimos, cosechar la semilla de sus mazorcas. Se espera que si cualquiera de los métodos para hacer sintéticos se generaliza entre este tipo de agricultores, sus rendimientos de grano por hectárea serán superiores a los de sus variedades criollas.

En el método clásico de obtención de sintéticos (Allard, 1960), un grupo de líneas de alta aptitud combinatoria (generación 0) se cruzan entre sí en todas las formas posibles, se siembra un compuesto balanceado formado con la semilla de las cruzas (generación 1), y de las plantas resultantes (generación 2) se cosecha la semilla del sintético en la cual se puede calcular su endogamia. Como la posterior producción masiva del sintético se lleva a cabo en poblaciones grandes, en éstas ya no se produce endogamia adicional. Sin embargo, Sahagún-Castellanos (1994) y Márquez-Sánchez (2011) encontraron que el sintético puede obtenerse en la primera generación, al dejar que el compuesto balanceado de las líneas o familias involucradas se polinice libremente en una parcela aislada. Además, aunque el compuesto no llegase en una sola generación al equilibrio, en las posteriores siembras masivas (con miles de plantas) ya no se generará endogamia.

\section{MATERIALES Y MÉTODOS}

Las familias de auto hermanos $(\mathrm{AH})$ o líneas $\mathrm{S}_{1}$ que forman el sintético son parte de un esquema de selección recurrente a largo plazo, que han sido seleccionadas de un experimento en el que se evalúan 200 familias, de las cuales se seleccionan 10 familias, cantidad equivalente a $5 \%$ del total. Como las familias seleccionadas provienen de la misma variedad, se espera que tengan floraciones simultáneas y que por tanto se recombinen entre sí aleatoriamente. Con la semilla remanente del primer ciclo de 
selección, las familias seleccionadas se someten a polinización libre, como se indica en los párrafos siguientes, en los que se ejemplifica con $n$ familias y $m$ plantas por familia. Desde luego que los sintéticos pueden generarse también con familias (líneas) $\mathrm{S}_{2}, \mathrm{~S}_{3}$, etc., pero en estos casos el problema es seleccionar a las sub líneas derivadas de $\mathrm{S}_{2}, \mathrm{~S}_{3}$, etc., lo cual puede hacerse mediante su comportamiento per se o bien, a través de su aptitud combinatoria general, lo que incrementaría el tiempo para obtener los sintéticos en uno y en dos años, respectivamente.

En la Figura 1 se muestra el esquema de un lote de polinización libre de familias de auto hermanos con $n^{2} m^{2}$ plantas, con $n$ familias y con un total de $n m^{2}$ familias. Cada apareamiento se indica primero con el número de la familia, enseguida con una coma y después con el número de la línea. El número de plantas en todas las familias es siempre el mismo $(m)$.

La Figura 2 muestra una familia, cada una con $m^{2}$ plantas; en la diagonal, de izquierda a derecha, hay $m$ plantas autofecundadas $(1,1 ; 2,2 ; 3,3 ; m, m)$, y las demás plantas se cruzan entre sí para producir $m(m-1)$ cruzas. Este diagrama se aplicaría para cada una de las 10 familias que se han supuesto.
Fuera de la diagonal están las familias no emparentadas que se cruzan con otras familias, cuyo número es $n(n-1)$. Si ahora se considera al lote en total, los respectivos números son: $n m, n m(n-1)$ y $n m^{2}(n-1)$. Si en éstos se utiliza al producto $n m$ como factor común, se obtienen los respectivos números codificados: $1, m-1$ y $m(n-1)$, los que divididos por el total $\mathrm{nm}$ generan las proporciones de las fuentes de endogamia que se muestran en el Cuadro 1.

Con el objeto de medir la endogamia, se tienen que calcular las coancestrías entre las plantas de cada grupo. Como se sabe, la coancestría es igual a la endogamia de la siguiente generación. Las respectivas coancestrías son: $\left(1+\mathrm{F}_{0}\right) / 2$, en donde $\left(1+\mathrm{F}_{0}\right) / 2=\left[\left(1+(1 / 2)\left(1+\mathrm{F}_{-1}\right)\right] / 2=1 / 2+(1+\right.$ $\left.\mathrm{F}_{-1}\right) / 4,\left(1+\mathrm{F}_{0}\right) / 2$ y la última es $\mathrm{F}_{1}$. Esta información se muestra concentrada en el Cuadro 1.

\section{RESULTADOS Y DISCUSIÓN}

La endogamia de la ecuación de selección recurrente, de acuerdo con la información del Cuadro 1, se obtiene con el promedio de la suma de los productos de número codificado x endogamia. El resultado para el sintético de auto hermanos, es el siguiente:

\begin{tabular}{|c|c|c|c|c|}
\hline & $\mathbf{1}$ & $\mathbf{2}$ & $\mathbf{3}$ & $\boldsymbol{n}$ \\
\hline $\boldsymbol{n}$ & $m$ & $m$ & $m$ & $m$ \\
\hline $\mathbf{1}$ & 1,1 & 1,2 & 1,3 & $1, n$ \\
\hline $\boldsymbol{m}$ & $m^{2}$ & $m^{2}$ & $m^{2}$ & $m^{2}$ \\
\hline $\mathbf{2}$ & 2,1 & 2,2 & 2,3 & $2, n$ \\
\hline $\boldsymbol{m}$ & $m^{2}$ & $m^{2}$ & $m^{2}$ & $m^{2}$ \\
\hline $\mathbf{3}$ & 3,1 & 3,2 & 3,3 & $3, n$ \\
\hline $\boldsymbol{m}$ & $m^{2}$ & $m^{2}$ & $m^{2}$ & $m^{2}$ \\
\hline $\boldsymbol{n}$ & $n, 1$ & $n, 2$ & $n, 3$ & $n, n$ \\
\hline $\boldsymbol{m}$ & $m^{2}$ & $m^{2}$ & $m^{2}$ & $m^{2}$ \\
\hline
\end{tabular}

Figura 1. Esquema de un lote de polinización libre de familias de auto hermanos para formar un sintético, con $n$ familias cada una con $m$ plantas.

\begin{tabular}{|c|c|c|c|c|}
\hline & $\mathbf{1}$ & $\mathbf{2}$ & $\mathbf{3}$ & $\boldsymbol{m}$ \\
\hline $\mathbf{1}$ & 1,1 & 1,2 & 1,3 & $1, m$ \\
\hline $\mathbf{2}$ & 2,1 & 2,2 & 2,3 & $2, m$ \\
\hline $\mathbf{3}$ & 3,1 & 3,2 & 3,3 & $3, m$ \\
\hline $\boldsymbol{m}$ & $m, 1$ & $m, 2$ & $m, 3$ & $m^{2}$ \\
\hline
\end{tabular}

Figura 2. Esquema de los cruzamientos en una familia con $m^{2}$ plantas. 


$$
\begin{aligned}
\mathrm{F}(\mathrm{AH})_{2} & =\left\{(1 / 2)+\left(1+\mathrm{F}_{-1}\right) / 4+\left[(m-1)\left(1+\mathrm{F}_{0}\right) / 2\right]+m(n-1) \mathrm{F}_{1}\right\} / n m \\
& =(1 / 4 n m)\left[2+1+\mathrm{F}_{-1}+2 m+2 m \mathrm{~F}_{0}-2-2 \mathrm{~F}_{0}+4 m(n-1) \mathrm{F}_{1}\right] \\
& =(1 / 4 n m)\left[(2 m+1)+4 m(n-1) \mathrm{F}_{1}+2(m-1) \mathrm{F}_{0}+\mathrm{F}_{-1}\right]
\end{aligned}
$$

Para obtener la endogamia del sintético en la segunda generación, como $\mathrm{F}_{1}=\mathrm{F}_{0}=\mathrm{F}_{-1}=0$, la Ec. 1 se transforma en:

$$
\mathrm{F}(\text { Sintético de } \mathrm{AH})_{2}=(1 / 4 n m)(2 m+1)
$$

[Ec. 2]

En la práctica los sintéticos se hacen con números menores de familias y de plantas por familia. En el Cuadro 2 se presentan ejemplos del Sint $\left(\mathrm{F}_{1}\right)$ con números de familias y de plantas por familia desde 10 hasta 50 , donde también se puede apreciar que el número de familias influye más en la endogamia que el número de plantas por familia.

En la información del Cuadro 2 se nota que la mayor endogamia del sintético $\mathrm{F}_{2}$ se genera con los menores tamaños de familias y de plantas por familia $(n=m=10)$ con endogamia igual a 0.0526, ya que Márquez-Sánchez (1979) encontró, al aplicar el método clásico para obtener un sintético (Allard, 1960), que eran suficientes ocho líneas autofecundadas; desde luego, con números mayores de familias y de plantas por familia la endogamia es menor, por ejemplo con $n=m=50$ la endogamia es la quinta parte de la generada $\operatorname{con} n=m=10$. En otras palabras, para obtener la menor endogamia deben usarse los mayores números de familias y de plantas por familia. Finalmente, los números de familias y de plantas por familia dependerán de la cantidad de semilla que se desé obtener del sintético.

\section{CONCLUSIONES}

Al aplicar el primer ciclo de selección de una ecuación recurrente de familias de auto hermanos, con las familias seleccionadas, en una sola etapa, se puede generar un sintético cuya endogamia, con 10 familias y 10 plantas por familia, es igual a 0.0526. Para obtener valores menores de endogamia pueden usarse mayores números de familias (líneas), aunque esto puede causar una reducción en el rendimiento del sintético.

\section{BIBLIOGRAFÍA}

Allard R W (1960) Principles of Plant Breeding. Wiley, New York. N. Y. 485. Márquez-Sánchez F (1979) An empirical approach for the prediction of $\mathrm{F}_{2}$ synthetics with varying numbers of lines. Crop Sci. 19:439 444.

Márquez-Sánchez F (2011) A corrected calculation of inbreeding in mass selection. Maydica 53:227-229.

Sahagún-Castellanos J (1994) Sobre el cálculo de la endogamia de variedades sintéticas. Agrociencia S. Fitociencia 5:67-68.

Cuadro 1. Fuentes de endogamia, números de plantas, números codificados de plantas y coancestrías (endogamia).

\begin{tabular}{lccc}
\hline Fuentes & Número de plantas & $\begin{array}{c}\text { Números codificados } \\
\text { de plantas }\end{array}$ & Endogamia \\
\hline $\begin{array}{l}\text { Autofecundaciones } \\
\begin{array}{l}\text { Cruzas entre plantas de la misma } \\
\text { autofecundación }\end{array}\end{array}$ & $n m(m-1)$ & 1 & $1 / 2+\left(1+\mathrm{F}_{-1}\right) / 4$ \\
$\begin{array}{l}\text { Cruzas entre plantas de } \\
\text { diferentes autofecundaciones }\end{array}$ & $n m^{2}(n-1)$ & $m-1$ & $\left(1+\mathrm{F}_{0}\right) / 2$ \\
\hline
\end{tabular}

Cuadro 2. Ejemplos de la endogamia ${ }^{\dagger}$ en sintéticos de auto hermanos con $n$ líneas y $m$ plantas por línea.

\begin{tabular}{lccccc}
\hline \multirow{2}{*}{$\begin{array}{l}\text { Número de plantas } \\
\text { por línea }(m)\end{array}$} & \multicolumn{5}{c}{ Número de líneas $(n)$} \\
\cline { 2 - 6 } 10 & 10 & 20 & 30 & 40 & 50 \\
\hline 20 & 0.0526 & 0.0263 & 0.0175 & 0.0131 & 0.0105 \\
30 & 0.0513 & 0.0256 & 0.0171 & 0.0128 & 0.0102 \\
40 & 0.0509 & 0.0254 & 0.0169 & 0.0127 & 0.0101 \\
50 & 0.0506 & 0.0253 & 0.0168 & 0.0126 & 0.0101 \\
\hline${ }^{\dagger}$ Los valores endogámicos iguales son aproximaciones. & 0.0505 & 0.0252 & 0.0168 & 0.0126 & 0.0101 \\
\hline
\end{tabular}

